\title{
Bildung für das Lehramt Chemie in Deutschland. Didaktik bereits im Studium
}

\author{
Michael W. Tausch*
}

Abstract: This article describes the training and further education of chemistry teachers in Germany. Specific features are highlighted and, in part, critically examined.

Keywords: Bildung Deutschland

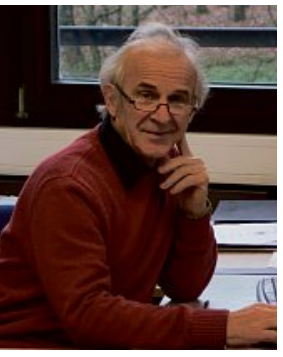

Michael W. Tausch studied chemistry at the Polytechnic Institute of Bucharest, Romania, from 1967 to 1972 . He subsequently studied mathematics and educational sciences in Bremen and Oldenburg, both Germany, and received his $\mathrm{PhD}$ from the University of Bremen in 1981. He was a teacher for chemistry and mathematics from 1976 to 1996. In 1996, he completed his habilitation at the University of Duisburg-Essen, Germany, and became Professor for Chemistry and Chemical Education there. In 2005, he moved to the Department for Chemistry Didactics at Bergische Universität Wuppertal, Germany.

He has published more than 250 papers and textbooks. His scientific work includes curriculum innovation by the development of experimental approaches and teaching materials on silicones, cyclodextrins and other advanced materials. He is performing pioneering work for the introduction and exploitation of photoprocesses in chemistry education. In $2015 \mathrm{M}$. W. Tausch was awarded the inaugural Heinz Schmidkunz Prize by the Gesellschaft Deutscher Chemiker (GDCH, German Chemical Society)

\section{Zweifachstudium in Chemie $+X$}

Das Lehramt Chemie muss in allen deutschen Bundesländern an einer Universität studiert werden. Allerdings bildet Baden-Württemberg eine Ausnahme: Hier studieren angehende Grund-, Hauptund Realschullehrerinnen und -lehrer an Pädagogischen Hochschulen.

Lehrkräfte mit regulärer Laufbahn (Lehramtsstudium und Referendariat) vertreten zwei Unterrichtsfächer. Entsprechend enthält das Lehramtsstudium für Chemie - trotz einiger landesspezifischer Unterschiede - an allen Hochschulen auch ein zweites Fach $\boldsymbol{X}$ und unterschiedliche Anteile an Erziehungswissenschaften $\boldsymbol{E} \boldsymbol{W}$. Das in Kombination mitChemie studierbare Zweitfach $\mathrm{X}$ ist in einigen Bundesländern, z.B. in Nordrhein-Westfalen, beliebig wählbar, in anderen, z. B. in Bayern, kann es nur mit bestimmten Fächern kombiniert werden. Wiederum bundeseinheitlich ist mittlerweile die Gliederung des Studiums in einen sechssemestrigen Kombi-BA (Kombinatorischer Bachelor of Arts) und einen viersemestrigen MEd (Master of Education). Diese Studienstruktur wurde im Zuge der Umsetzung des BolognaProzesses eingeführt. Sie ersetzt die frühere Studienstruktur nach Landesprüfungsordnungen LPO, zu deren Merkmalen eine Zwischenprüfung und ein erstes Staatsexamen gehörten. Der KombiBA und der MEd bestehen aus Modulen im Fach Chemie, dem Zweitfach $\mathrm{X}$ und Erziehungswissenschaften mit jeweils festgelegten credit points, workloads und Prüfungsleistungen. Der Abschluss Master of Education ersetzt das frühere erste Staatsexamen und macht den Schritt ins Referendariat frei. In Bayern muss zusätzlich zum MEd auch ein erstes Staatsexamen an der Universität bestanden werden, um den Eintritt ins Referendariat zu erlangen.

Das deutsche Spezifikum des ZweiBergische Universität Wuppertal Fakultät 4, Chemie, Gaußstr. 20 D-42119 Wuppertal

E-Mail: mtausch@uni-wuppertal.de
fach-Lehrers mag finanziell und verwaltungstechnisch für die Ministerien und
Schulbehörden vorteilhaft sein. Was aber die Breite und Tiefe anbetrifft, in der das Fach Chemie studiert werden kann, verursacht dieses Studienformat im Vergleich zu den Einfach-Chemielehrern aus anderen Europäischen Ländern, z. B. der Schweiz, erhebliche Defizite. Es erübrigt sich, im Detail darauf einzugehen und zu begründen, dass in einer Studienzeit von zehn Semestern beim Studium zweier Fächer für jedes der beiden Fächer weniger ,herüberkommt", als beim Vollstudium des einen Faches.

\section{Fachinhalte und Didaktik im Studium}

Bei der Umstellung der Studienstruktur nach den Bologna-Prozess-Vorschlägen bestand die Gefahr, dass die fachwissenschaftlichen Anteile des Lehramtsstudiums Chemie noch stärker schrumpfen könnten. Die „Fachgruppe Chemieunterricht" der Gesellschaft Deutscher Chemiker hatte seinerzeit Vorschläge für die Strukturierung der Curricula im BachelorMaster-Studium Lehramt Chemie erarbeitet. Danach sollten die Grundmodule in Chemie für die Lehramts- und Fachstudierenden gemeinsam angeboten werden. Gleichwohl müssen eine fachwissenschaftliche Stoffauswahl gefunden und Schwerpunkte gesetzt werden, um das Zeitbudget für das Zweifachstudium einhalten zu können. Bei den in Abb. 1 angegebenen Studieninhalten sollte exemplarischen Vertiefungen der Vorrang vor einer allzu breiten Stoffvermittlung gegeben werden. ${ }^{[1]}$

Das charakteristische Merkmal in der Chemielehrerausbildung und in der Lehrerausbildung schlechthin ist die Fachdidaktik. Sie ergänzt die fachwissenschaftliche Ausbildung mit dem Fokus auf die angestrebte Lehrertätigkeit. Die Inhalte und Methoden in den Didaktik-Modulen lehnen sich eng an die fach- und bildungswissenschaftliche Ausbildung an und ver- 


\begin{tabular}{|c|c|}
\hline Studium für LA Sek I & Erweitert im Studium für LA an Cym / Sek II \\
\hline \multicolumn{2}{|c|}{ Allgemeine anorganische und analytische Chemie } \\
\hline $\begin{array}{l}\text { - Chemie der Hauptgruppen } \\
\text { - Chemie wässriger Lösungen } \\
\text { - Molekül- und Festkörperchemie } \\
\text { - Methoden der analytischen Chemie } \\
\text { - Synthetische Methoden / industr. } \\
\text { Anwendungen } \\
\text { - Atome und Atomverbände } \\
\text { - Struktur-Eigenschaftsbeziehungen }\end{array}$ & $\begin{array}{l}\text { - Größerer Vertiefungsgrad der für Sek. I genannten Inhaltsbereiche, } \\
\text { dazu: } \\
\text { - Nebengruppenelemente, } \\
\text { Koordinationschemie } \\
\text { - Spektroskopie } \\
\text { - Bioanorganische Chemie } \\
\text { - Metallorganische Chemie, } \\
\text { homogene Katalyse } \\
\text { - Synthesemethoden } \\
\text { - Spezielle spektroskopische Methoden }\end{array}$ \\
\hline \multicolumn{2}{|c|}{ Organische und biologische Chemie } \\
\hline $\begin{array}{l}\text { - Stoffklassen, funktionelle Gruppen } \\
\text { - Ausgewählte Reaktionsmechanismen und Synthesen } \\
\text { - UV / Vis- und IR-Spektroskopie } \\
\text { - Natürliche und synthetische Makromoleküle } \\
\text { - Farbstoffe und Färbeverfahren } \\
\text { - Grundlagen des Stoff- und Energiewechsels } \\
\text { - Struktur-Eigenschaftsbeziehungen } \\
\text { - Nachhaltigkeit als Grundprinzip chemischer For- } \\
\text { schung und Produktion }\end{array}$ & $\begin{array}{l}\text { - Größerer Vertiefungsgrad der für Sek. I genannten Inhaltsbereiche, } \\
\text { dazu: } \\
\text { - Isomerie und Stereochemie } \\
\text { - Reaktionsmechanismen und } \\
\text { Zwischenstufen } \\
\text { - Grundlagen der Photochemie } \\
\text { - Aromaten und Heterocyclen } \\
\text { - Synthese und Katalyse } \\
\text { - Biopolymere, Coenzyme, Naturstoffe } \\
\text { - Grundlagen der NMR-Spektroskopie und der Massenspektroskopie }\end{array}$ \\
\hline \multicolumn{2}{|c|}{ Physikalische Chemie } \\
\hline $\begin{array}{l}\text { - Energie und Entropie } \\
\text { - Reaktionsgeschwindigkeit } \\
\text { - Elektrochemie }\end{array}$ & $\begin{array}{l}\text { - Größerer Vertiefungsgrad der für Sek. I genannten Inhaltsbereiche, } \\
\text { dazu: } \\
\text { - Makroskopische Eigenschaften der Stoffe (Hauptsätze der Thermo- } \\
\text { dynamik, Phasengleichgewichte Chemisches Gleichgewicht) } \\
\text { - Mikroskopische Struktur der Materie (Atome, Moleküle, Molekül- } \\
\text { spektroskopie, Statistik) } \\
\text { - Kinetik und Dynamik chemischer Reaktionen (Reaktionsgeschwin- } \\
\text { digkeit, Reaktionsmechanismen, Transporteigenschaften) } \\
\text { - Kinetische Gastheorie }\end{array}$ \\
\hline \multicolumn{2}{|r|}{ Chemiedidaktik } \\
\hline \multicolumn{2}{|c|}{$\begin{array}{l}\text { - Fachdidaktische Reflexion von Basiskonzepten der Chemie } \\
\text { - Fachdidaktische Forschung und Positionen } \\
\text { - Konzeptionen und Curricula } \\
\text { - Lernprozesse, Diagnose von Lernschwierigkeiten, Motivation und Interesse } \\
\text { - Schulrelevante Aspekte der Geschichte der Chemie } \\
\text { - Nachhaltige Entwicklung } \\
\text { - schulorientiertes Experimentieren } \\
\text { - Praxisphasen mit dem Ziel der Erkundung und Analyse schulischer und } \\
\text { außerschulischer Berufffelder Einschließlich Vor- und Nachbereitung }\end{array}$} \\
\hline
\end{tabular}

Abb. 1. Studieninhalte - Empfehlungen der GDCh-Fachgruppe Chemieunterricht. ${ }^{[1]}$

mitteln für die Praxis des Chemieunterrichts relevante Kenntnisse und Kompetenzen (Stichwörter: schulorientiertes Experimentieren, forschend-entwickelnde Vorgehensweise, Basiskonzepte, Planen von Unterrichtseinheiten, konstruktivistische Lernschleifen, interdisziplinäre Themenfelder, digitale Medien, curriculare Innovation).

\section{Referendariat}

Das Referendariat gilt als Vorbereitungsdienst für die Laufbahn einer beamteten Lehrkraft. Hier erteilen die Lehramtsanwärter eigenverantwortlichen Unterricht in beiden Unterrichtsfächern, allerdings in reduziertem Maß. Einige

Unterrichtsstunden werden bei UnterVordergrund. der Bundesländer als Basiskonzepte implementiert wurden. Sie werden im Kernlehrplan für Chemie in NordrheinWestfalen $^{[3]}$ folgendermaßen beschrieben: „Basiskonzepte sind grundlegende, für den Unterricht eingegrenzte und für Schülerinnen und Schüler nachvollziehbare Ausschnitte fachlicher Konzepte und Leitideen. Sie stellen elementare Prozesse, Gesetzmäßigkeiten und Theorien dernaturwissenschaftlichen Fächer strukturiert und vernetzt dar. Sie beinhalten zentrale, aufeinander bezogene naturwissenschaftliche Begriffe, erklärende Modellvorstellungen und Theorien, die sich in dem jeweiligen Fach zur Beschreibung elementarer Phänomene und Prozesse als relevant herausgebildet haben. Dabei erheben sie nicht den Anspruch, jeweils das gesamte Fach vollständig abzubilden. Die besondere Bedeutung der Basiskonzepte für das Lernen besteht darin, dass mit ihrer Hilfe schulische Inhalte der einzelnen naturwissenschaftlichen Fächer sinnvoll strukturiert werden und die fachlichen Beziehungen durch den Konzeptgedanken über die gesamte Lernzeit miteinander verbunden werden können".

Unter den Zwängen des beschränkten Stundenkontingents für den Chemieunterricht und des föderalen Bildungssystems in Deutschland sind die fünf Basiskonzepte aus Tabelle 1 durchaus sinnvoll und für die Strukturierung von wichtigen Segmenten des Chemieunterrichts tauglich.

Es gibt aber auch einige weitere Schlüsselkonzepte [4], die bei der Vermittlung von Chemie den fünf Basiskonzepten aus Tabelle 1 sogar überzuordnen wären, beispielsweise:

- Arbeitsweisen und Erkenntniswege (Experimente, Hypothesen etc.),

- Denken und Argumentieren in Gegensatzpaaren (hydrophil-hydrophob, polar-unpolar, exotherm-endotherm, nukleophil-elektrophil, AnalyseSynthese, Homolyse-Heterolyse, Addition-Eliminierung, AbsorptionEmission etc.) richtsbesuchen durch die Fachleitung des Studienseminars und die Schulleitung diskutiert und mit Noten bewertet. Das Referendariat dauert in der Regel anderthalb Jahre und wird mit dem Staatsexamen abgeschlossen. Ein besonderes Gewicht wird in Studienseminaren auf die Einhaltung der Vorgaben durch die jeweils gültigen Lehrpläne gelegt. Im Fach Chemie stehen dabei die Kontextund Kompetenzorientierung sowie die Basiskonzepte des Chemieunterrichts im

Diesbezüglich ist positiv hervorzuheben, dass die Kultusministerkonferenz KMK sich auf bundeseinheitliche Bildungsstandards fürden Chemieunterricht ${ }^{[2]}$ einigen konnte, die in den Lehrplänen
- Klasseneinteilungen und Ordnungsrelationen (Element- und Stofffamilien, Redoxpotenziale, Säurestärken etc.)

- Nachhaltigkeit im Umgang mit Stoffen und Verfahren (Recycling, produktionsintegrierter Umweltschutz etc.).

Insofern gehören die fünf Basiskonzepte ebenso wie die obligatorischen Inhalte aus den Lehrplänen von Zeit zu Zeit auf den Prüfstand. Sie müssen am jeweils aktuellen Stand der wissenschaftlichen Erkenntnisse, alltagsrelevanten technischen Anwendungen und didaktischen Prägnanz in der Vermittlung von Chemie gemessen und gegebenenfalls nachjustiert werden. ${ }^{[4]}$ 
Tabelle 1. Fünf Basiskonzepte für den Chemieunterricht nach den KMK-Bildungsstandards

Basiskonzepte in der S
- Stoff-Teilchen
- Struktur-Eigenschaft
- Chemische Reaktion
- Energie

in der Sekundarstufe I
Basiskonzepte in der Sekundarstufe II

- Stoff-Teilchen

- Struktur-Eigenschaft

- Donator-Akzeptor

- Gleichgewicht

- Energie

\section{Lehrerfortbildung}

Die Chemie ist eine Disziplin, die sich rasant entwickelt. Wer seine Schüler für Chemie begeistern will, kann nicht von seiner Verbeamtung bis zur Pensionierung nur mit dem punkten, was er im Studium und im Referendariat erworben hat. Fortbildung als „lebenslanges Lernen“ ist notwendig, ganz gleich ob es sich um die Zweifach-Chemielehrerin nach deutschem Muster oder den Einfach-Chemielehrer nach dem Ausbildungsmuster in der Schweiz handelt.

In Deutschland wird auf Länderebene Lehrerfortbildung von Kompetenzzentren koordiniert und von Teams aus engagierten Lehrkräften durchgeführt. Die Fachgruppe Chemieunterricht der GDCh koordiniert und finanziert sieben Lehrerfortbildungszentren in Frankfurt, Leipzig,
Rostock, Bremen, Dortmund, Karlsruhe und Nürnberg. Diese bieten vorwiegend für die Lehrkräfte aus der jeweiligen Region, aber auch überregional und im Ausland verschiedene Fortbildungsthemen an, die man auf ihren Websites finden kann. Einige Didaktik-Lehrstühle von deutschen Hochschulstandorten, außerhalb der GDCh-Zentren, beispielsweise Freiburg, Wuppertal, Köln, Potsdam, München und Münster, sind ebenfalls sehr aktiv in der Lehrerfortbildung. Sie haben dazu Experimentier- und Materialiensets entwickelt, die sie in den Workshops bei den Lehrerfortbildungskursen einsetzten und als kompakte Koffer oder in Plastikboxen zu relativ günstigen Preisen den Schulen zur Verfügung stellen. Die beiden Koffer aus Abb. 2 wurden in Wuppertal und Köln entwickelt. Details dazu sind online über die Links in Lit. [5] und [6] zugänglich.

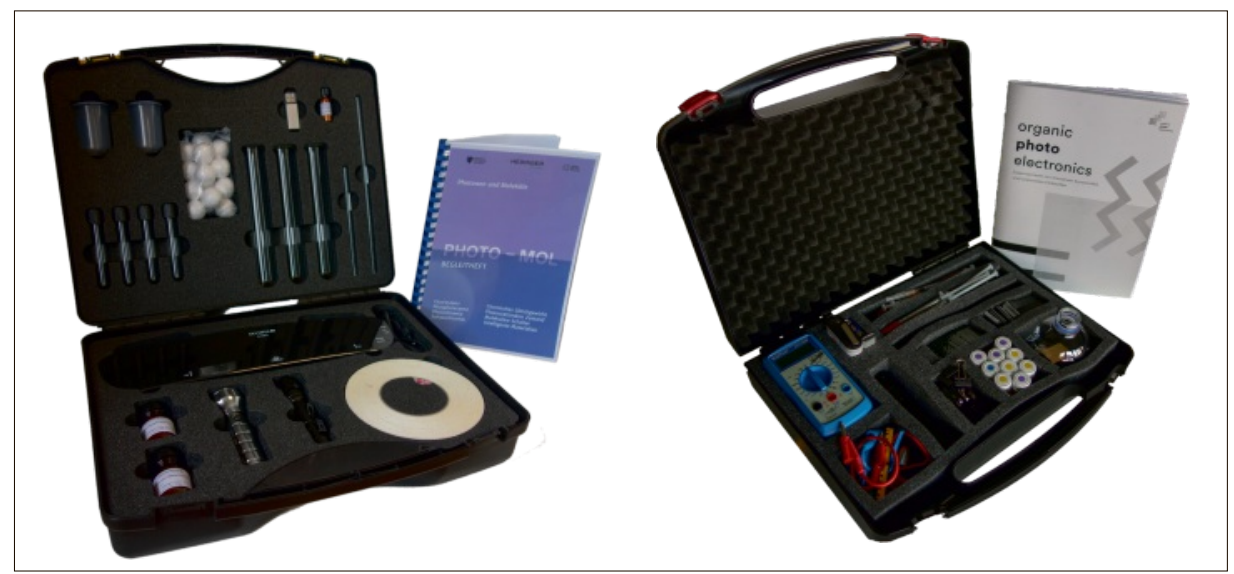

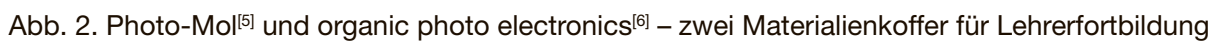
und Unterricht.

\section{Fazit}

Trotz der beschränkten fachwissenschaftlichen Anteile im Lehramtsstudium, das außer Chemie noch ein zweites Fach und Erziehungswissenschaften beinhaltet, bestehen für Chemie-Lehrkräfte in Deutschland durch die fachdidaktischen Anteile im Studium und das Referendariat reelle Chancen, über die gesamte Zeit ihrer Lehrtätigkeit guten Chemieunterricht zu erteilen. Eine wesentliche Voraussetzung dafür ist, sich kontinuierlich fortzubilden und regelmäßig an Lehrerfortbildungskursen teilzunehmen.

\section{Danksagung}

Der Deutschen Forschungsgemeinschaft DFG wird für die Förderung der Forschungsvorhaben TA 228/4-1 Photo-LeNa (Photoprozesse in der Lehre der Naturwissenschaften, 2013-2016) und TA 228/4-2 Photo-MINT (Photoprozesse in den MINTFächern, 2017-2020) gedankt.

Received: October 19, 2017

[1] GDCh, Fachgruppe Chemieunterricht, Das Bachelor-/Master-Studiumfür das Lehramt Chemie, Vorschläge zur Restrukturierung und zur curricularen Entwicklung, Frankfurt a.M., 2008.

[2] Sekretariat der Kultusministerkonferenz (Hrsg.), 'Bildungsstandards Chemie', Luchterhand Verlag, München, 2005.

[3] Düsseldorf (Hrsg.), 'Kernlehrplan Chemie Gymnasium Sek. I in Nordrhein-Westfalen', Ritterbach Verlag Frechen, 2008.

[4] M. W. Tausch, 'Chemische Schlüsselkonzepte - Netzwerk aus Leitideen für Unterricht und Lehre', Praxis der Naturwissenschaften Chemie in der Schule, 2017, 66 (1), 5; dieser Artikel kann online unter http://www.chemiedidaktik.uni-wuppertal.de/publikationen/index. $h t m l$ aufgerufen werden.

[5] M. W. Tausch, N. Meuter, 'Funktionelle Farbstoffe - Interaktionsbox für Schulen und Universitäten', Praxis der Naturwissenschaften - Chemie in der Schule, 2016, 65 (1), 5; http:// www.chemiedidaktik.uni-wuppertal.de/lehre/ photo-mol/index.html

[6] M. W. Tausch, J. Dörschelln, A. Banerji, M. Zepp, 'organic photo electronics - Didaktisches Kofferset zu organischen LEDs und Solarzellen', Praxis der Naturwissenschaften - Chemie in der Schule, 2016, 65 (1), 28; https://docs.google.com/forms/d/e/1 FAIpQLSc9GNzjko3O4-l8rBrFe5ov8gWbnWerNK59iIa4cQqrwNeNMw/ viewform 